\title{
The impact of immunosenescence on the efficacy of immune checkpoint inhibitors in melanoma patients: a meta-analysis
}

This article was published in the following Dove Press journal:

OncoTargets and Therapy

Ping $\mathrm{Li}^{\prime}$

Xuefang Yang ${ }^{2}$

Yumiao Feng'

Lijuan $\mathrm{Wu}^{\prime}$

Wei $\mathrm{Ma}^{\prime}$

Gaozhong Ding'

Yun Wei'

Lan Sun'

'Department of Dermatology, The Affiliated Suzhou Hospital of Nanjing Medical University, Jiangsu 21500 I, China; ${ }^{2}$ The Affiliated Suzhou Hospital of Nanjing Medical University, Suzhou Municipal Hospital Eastern, Jiangsu 2I500I, China
Correspondence: Lan Sun

Department of Dermatology, The Affiliated Suzhou Hospital of Nanjing Medical University, 16 Baitaxi Road, Gusu District, Suzhou 21500I, Jiangsu, China. Email lansunnjmu@yandex.com
Background: Immunosenescence, the age-related decline of immunity, affects the immune responses of melanoma patients. Through immune responses, immune checkpoint inhibitors (ICIs) exert their antitumor robustness. In different ages of melanoma patients, especially the older patients, the effectiveness of ICIs remains unclear. It is still controversial whether ICIs should be used in treating older patients.

Materials and methods: The authors included clinical trials of ICIs in older and younger patients. The authors used hazard ratio (HR) and 95\% CI of overall survival (OS).

Results: From four phase III randomized clinical trials 2,251 melanoma patients were included. We found that ICIs significantly prolonged the OS for melanoma patients in both younger (HR, $0.71 ; 95 \%$ CI, $0.60-0.82 ; P<0.001$ ) and older groups (HR, 0.62; 95\% CI, $0.41-0.83 ; P<0.001$ ) compared with controls. Anti-programmed death-1 (PD-1) agents appeared to be more efficient in older melanoma patients (HR, $0.34 ; 95 \% \mathrm{CI}, 0.14-0.53)$ versus younger patients (HR, 0.52 ; 95\% CI, 0.26-0.78).

Conclusion: ICIs significantly prolonged the OS for melanoma patients in both younger and older groups than controls. Anti-PD-1 agents were more efficient in older melanoma patients versus younger patients. ICIs could be used for older melanoma patients.

Keywords: immunosenescence, aging, immune checkpoint inhibitor, PD-1, melanoma

\section{Introduction}

Immunosenescence is a phenomenon of gradual deterioration of the immune system with increasing age. ${ }^{1}$ It involves both the host's capacity to respond to infections and the development of long-term immune memory. It was observed that immune status altered in aged people ${ }^{1}$ and immunosenescence even remodel the immune system, hence inducing numbers of diseases. ${ }^{2}$ Previous researches manifested that immunosenescence can change the adaptive immune response and induce neuroinflammatory diseases. ${ }^{3}$ Aging of hematopoietic stem cell compartment, alteration of antigen-presenting cell functions, and reduced antitumor ability of $\mathrm{T}$ cells were all associated with increasing age. ${ }^{4}$ Immunosenescence may cause tissue and cell damages and immunosurvey escape, thereby resulting in tumor development. However, in many fields, the impact of immunosenescence on the efficacy of some drugs still remains unclear.

Immune checkpoint inhibitors (ICIs), including programmed death-1 (PD-1)/PD-L1 inhibitors and cytotoxic T-lymphocyte-associated antigen 4 (CTLA-4) inhibitors, are novel antitumor drugs that have brought hope to cancer patients, especially the nonsmall cell lung cancer (NSCLC) patients and melanoma patients. ${ }^{5,6}$ The monotherapy 
and combination immunotherapy of ICIs have been applied in the clinical practice. ${ }^{7}$ They were proved to be more efficient and generate less toxicity in cancer treatment, making them a very attractive therapeutic option. ${ }^{8}$ It is currently well accepted by the clinicians that overall response rate, overall survival (OS), and progression-free survival (PFS) can be significantly improved in melanoma patients. ${ }^{8}$ Several clinical trials have observed the impact of immunosenescence on the effectiveness of ICIs. Aged patients may benefit less from the PD-1 inhibitors and CTLA-4 inhibitors in certain cancers. ${ }^{4}$

Two groups have reported that increasing age can influence the efficacy of PD-1 inhibitors in lung cancer treatment. ${ }^{9}$ However, among melanoma patients belonging to different age groups, it still remains unclear whether CTLA-4 or PD-1 inhibitors are recommended. This topic is of importance considering the efficacy of ICI treatment and the increasing number of ICI application in daily clinic life. Differences in response to ICI in younger and older patients would have serious influence on clinical applicability, therapy recommendations, and treatment guidelines. We hypothesize that effectiveness of ICIs in melanoma patients may vary in different age groups. Hence, based on previous clinical trials, we conducted a meta-analysis that was particularly focused on melanoma patients belonging to different age groups.

\section{Materials and methods}

\section{Study design and search strategy}

This meta-analysis was based on data from randomized clinical trials (RCTs), which compared ICIs with controls in younger and older melanoma patients. We searched for trials on PubMed/Medline, Embase, the Cochrane Central Register of Controlled Trials (CENTRAL), and Google Scholar; January 2018 was the cutoff date. Keywords included ICI, age, and clinical trial. All the studies of potential interest were retrieved.

\section{Selection criteria}

Eligible studies should fulfill the following criteria: 1) trials that included patients with melanoma, 2) interventions of ICIs, 3) trials that compared ICIs and controls, 4) trials that reported hazard ratios (HRs) of OS or PFS and subgroup analysis by age, and 5) phase III randomized clinical trials. All the papers in non-English language were excluded.

\section{Data extraction}

Two reviewers (PL and LS) independently extracted data by using an extraction form. The two reviewers checked all the data carefully. All studies were identified by first author and the year of publication. Information from the included studies was extracted after review, including publication year, inclusion/exclusion criteria, patient numbers, treatment in the experimental and control cohort, and HR of OS in younger and older groups.

\section{Risk of bias assessment}

Two reviewers (PL and LS) independently assessed the risk of bias using the Cochrane Collaboration tool. All disagreements were resolved by the first author (PL). Details on the risk of bias in eight studies are illustrated in Figure 1.

\section{Statistical analysis}

Statistical analysis, forest plots, and detection of publication bias were done with Stata SE (StataCorp, College Station, TX, USA). Risk of bias analysis was conducted using REVMAN (RevMan5.3; The Nordic Cochrane Centre, The Cochrane Collaboration, Copenhagen, Denmark). HRs were used for evaluation and 95\% CIs were calculated for each estimate. A cutoff $P$-value of 0.05 was considered statistically significant. Heterogeneity was considered low, moderate, or high for $I^{2}<25 \%, 25 \%-50 \%$, and $>50 \%$, respectively. If the $I^{2}$ value was lower than $50 \%$, a fixed model would be used; if the $I^{2}$ value was more than $50 \%$, a random model would

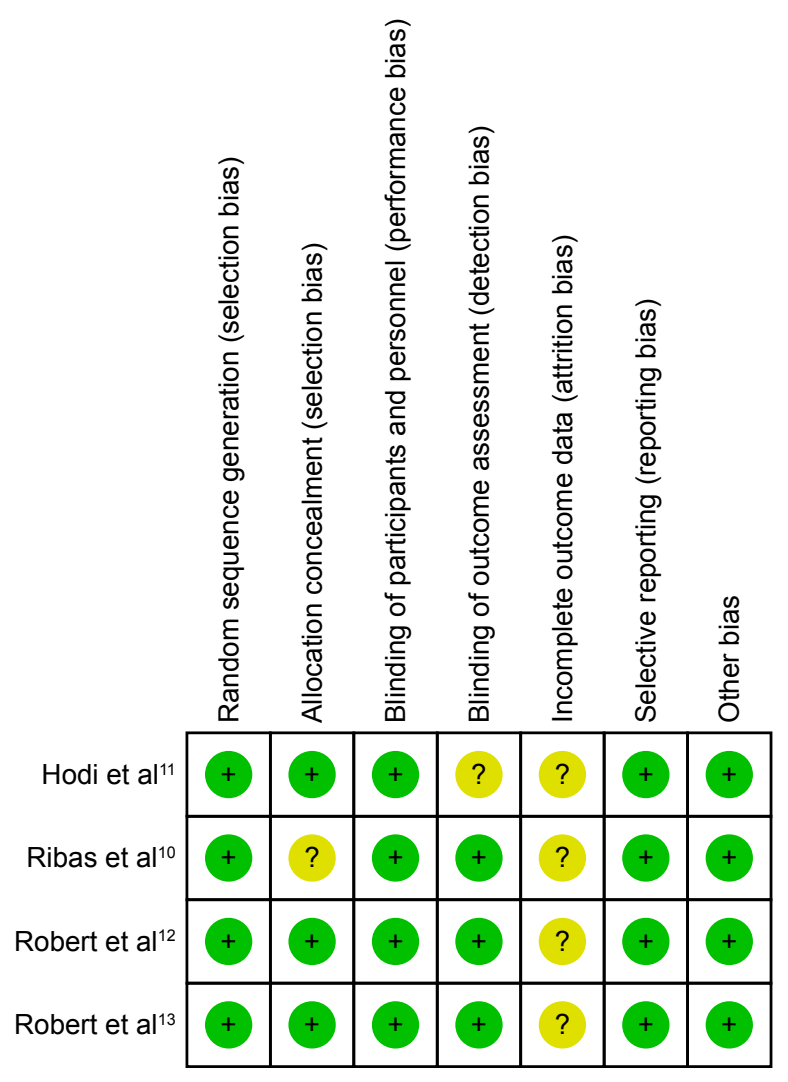

Figure I Risk of bias assessment of included studies. 
be used. Publication bias was assessed by both Begg's test and Egger's test.

\section{Results}

\section{Patient characteristics}

In all, 2,251 melanoma patients from four phase III RCTs were included in the analysis. ${ }^{10-13}$ The trial selection process is illustrated in Figure 2. All the trials were phase III RCTs. ${ }^{10-13}$ In all, 764 older patients (aged $\geq 65$ years) and 1,487 younger patients (aged $<65$ years) were included in the analysis. All the studies assessed the efficacy of ICIs including tremelimumab, ipilimumab, and nivolumab. The main characteristics of all the trials are available in Table 1.

\section{Comparison of OS in younger and older patients}

We first attempted to conduct the analysis of PFS; however, it was not further conducted due to the lack of available data.
One trial,${ }^{14}$ which was initially included in the analysis, was excluded because HR of OS was not reported. We used Stata 14 SE to perform the statistical analysis, and the summary HR and 95\% CI were calculated. A total of 2,251 melanoma patients from four trials were included in our analysis. ${ }^{10-13} \mathrm{We}$ found that ICIs significantly prolonged the OS for melanoma patients in both younger (HR, $0.71 ; 95 \%$ CI, $0.60-0.82 ; P<0.001)$ and older groups (HR, $0.62 ; 95 \%$ CI, $0.41-0.83 ; P<0.001$ ) compared with controls (Figure 3 and Table 1).

\section{Subgroup analysis of OS}

Depending on the heterogeneity, we used fixed-effects model and random-effects model in the younger $\left(I^{2}=32.5 \%\right)$ and older group $\left(I^{2}=68.9 \%\right)$, respectively. When treated with CTLA-4 inhibitors, both younger and older patients had significantly longer survival, and no significant difference was observed between the two groups $(P=0.739)$

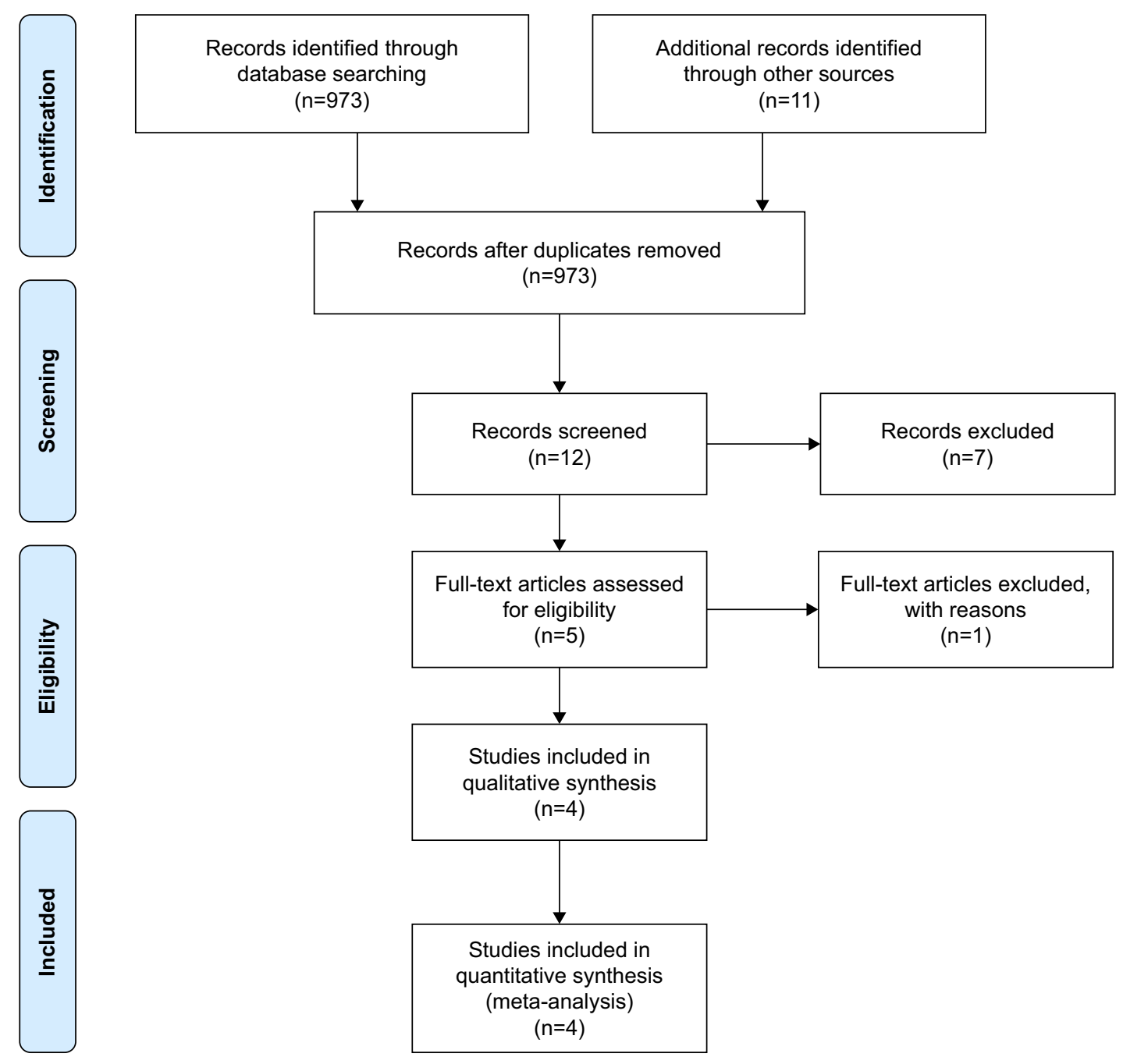

Figure 2 The screening process of included studies. 
Table I Subgroup analysis of hazard ratios for overall survival

\begin{tabular}{llll}
\hline Subgroup & Age & HR $(95 \% \mathrm{CI})$ & P-value \\
\hline CTLA-4 inhibitor & Younger & $0.74(0.64,0.84)$ & $<0.00$ I \\
& Older & $0.77(0.62,0.91)$ & $<0.00$ I \\
PD-I inhibitor & Younger & $0.52(0.26,0.78)$ & $<0.00$ I \\
& Older & $0.34(0.14,0.53)$ & 0.00 I \\
Subtotal & Younger & $0.71(0.60,0.82)$ & $<0.00$ I \\
& Older & $0.62(0.41,0.83)$ & $<0.00$ I \\
\hline
\end{tabular}

Note: The cutoff age was 65 years.

Abbreviations: CTLA-4, cytotoxic T-lymphocyte-associated antigen 4; HR, hazard ratio; PD-I, programmed death-I.

(Figures 4 and 5). Comparatively, anti-PD-1 agents appeared to be more efficient in older melanoma patients (HR, 0.34; 95\% CI, 0.14-0.53) versus younger patients ( $\mathrm{HR}, 0.52 ; 95 \%$ CI, 0.26-0.78), and this might result from a relatively small sample size. Notably, among older melanoma patients over 75 years treated with PD-1 inhibitors, a benefit of OS was significant $(P=0.001)$. Compared with younger patients, patients older than 75 years benefit more from melanoma, which is totally different from the situation in NSCLC treatment. These data will require further evaluation in future clinical trials. The difference of effectiveness of PD-1 and
CTLA-4 inhibitors in younger and older melanoma patients should get noticed in the future clinical practice.

\section{Publication bias analysis, sensitivity analysis, and risk of bias assessment}

A sensitivity analysis was conducted by recalculating the HR of different subgroups of studies based on relevant features, and no sensitivity analysis was positive (data not shown).

There was no publication bias based on the Begg's test and Egger's test for small-study effects. In Egger's test, the $P$-value was 0.803 . Likewise, in the Begg's test, the continuity-corrected $P$-value was 0.788 . This result indicated that no publication bias was detected in this study. The assessment of risk of bias is shown in Figure 1.

\section{Discussion}

This meta-analysis was the first study investigating the association between ages and survival of melanoma patients in immune checkpoint blockade treatment. Recent clinical trials suggested the potential impact of age on the efficacy of ICIs. Nevertheless, there is no final conclusion partly because of the lack of statistical power. We conducted this study in order to verify our hypothesis, and we attempted to provide possible practical suggestions in melanoma
References ID

\begin{tabular}{|c|c|c|}
\hline Ribas et $a^{10}$ & $\longrightarrow$ & $0.88(0.72,1.07)$ \\
\hline Ribas et $a^{10}$ & $\longrightarrow$ & $0.87(0.64,1.18)$ \\
\hline Hodi et $\mathrm{al}^{11}(\mathrm{Ipi}+\mathrm{gp} 100)$ & $\longrightarrow$ & $0.70(0.54,0.90)$ \\
\hline Hodi et al'11 (Ipi+gp100) & & $0.69(0.47,1.01)$ \\
\hline Hodi et al ${ }^{11}(\mid p i+p l b)$ & $\longrightarrow$ & $0.65(0.47,0.90)$ \\
\hline Hodi et al ${ }^{11}$ (Ipi+plb) & & $0.61(0.38,0.99)$ \\
\hline Robert et $\mathrm{al}^{12}$ & $\longrightarrow$ & $0.70(0.55,0.88)$ \\
\hline Robert et al ${ }^{12}$ & & $0.91(0.65,1.29)$ \\
\hline Robert et al $^{13}$ & $\longrightarrow$ & $0.52(0.32,0.85)$ \\
\hline Robert et $\mathrm{al}^{13}(65-75)$ & $\longrightarrow$ & $0.44(0.24,0.81)$ \\
\hline Robert et $\mathrm{al}^{13}(>75)$ & $\longrightarrow$ & $0.25(0.10,0.62)$ \\
\hline
\end{tabular}

Figure 3 Forest plots of HRs for OS.

Note: Random-effects model were used in the analysis.

Abbreviations: HR, hazard ratio; Ipi, ipilimumab; plb, placebo. 


\section{References ID}

Ribas et al ${ }^{10}$

Hodi et al ${ }^{11}($ Ipi+gp100)

Hodi et al11 (Ipi+plb)

Robert et $\mathrm{al}^{12}$

Robert et al ${ }^{13}(65-75)$

Robert et al ${ }^{13}(>75)$
HR $(95 \% \mathrm{Cl})$

$0.87(0.64,1.18)$

$0.69(0.47,1.01)$

$0.61(0.38,0.99)$

$0.91(0.65,1.29)$

$0.44(0.24,0.81)$

$0.25(0.10,0.62)$

$\begin{array}{llllllll}0 & 0.2 & 0.4 & 0.6 & 0.8 & 1 & 1.2 & 1.4\end{array}$

Figure 4 Subgroup analysis of older patients.

Note: Random-effects model were used in the analysis.

Abbreviations: HR, hazard ratio; Ipi, ipilimumab; plb, placebo.

treatments. We found that with a cutoff age of 65 years, ICIs significantly improved the OS and PFS in younger and older patients versus chemotherapy and gp100 vaccines. Interestingly, patients older than 75 years benefit more from melanoma compared with younger patients, which is totally different from the situation in NSCLC treatment. This observation was on the opposite with the possible impact of immunosenescence on the adaptive immune system. The observed results may not be entirely applicable to all patients treated in the community.
Immunosenescence refers to a physiological phenomenon causing alterations in the immune system with increasing age. In recent years, numbers of studies have focused on this field in order to elucidate the mechanism of immunosenescence, although a multitude of preclinical and clinical observations is lacking explanations. Immunosenescence will affect the function of dendritic cells, $\mathrm{CD} 4^{+} \mathrm{T}$ cells, $\mathrm{CD}^{+} \mathrm{T}$ cells, and regulatory T cells. ${ }^{15}$ CTLA-4 is focused on secondary lymphoid organs, while PD-1 inhibitors function majorly in peripheral tissues and the tumor microenvironment.
References ID

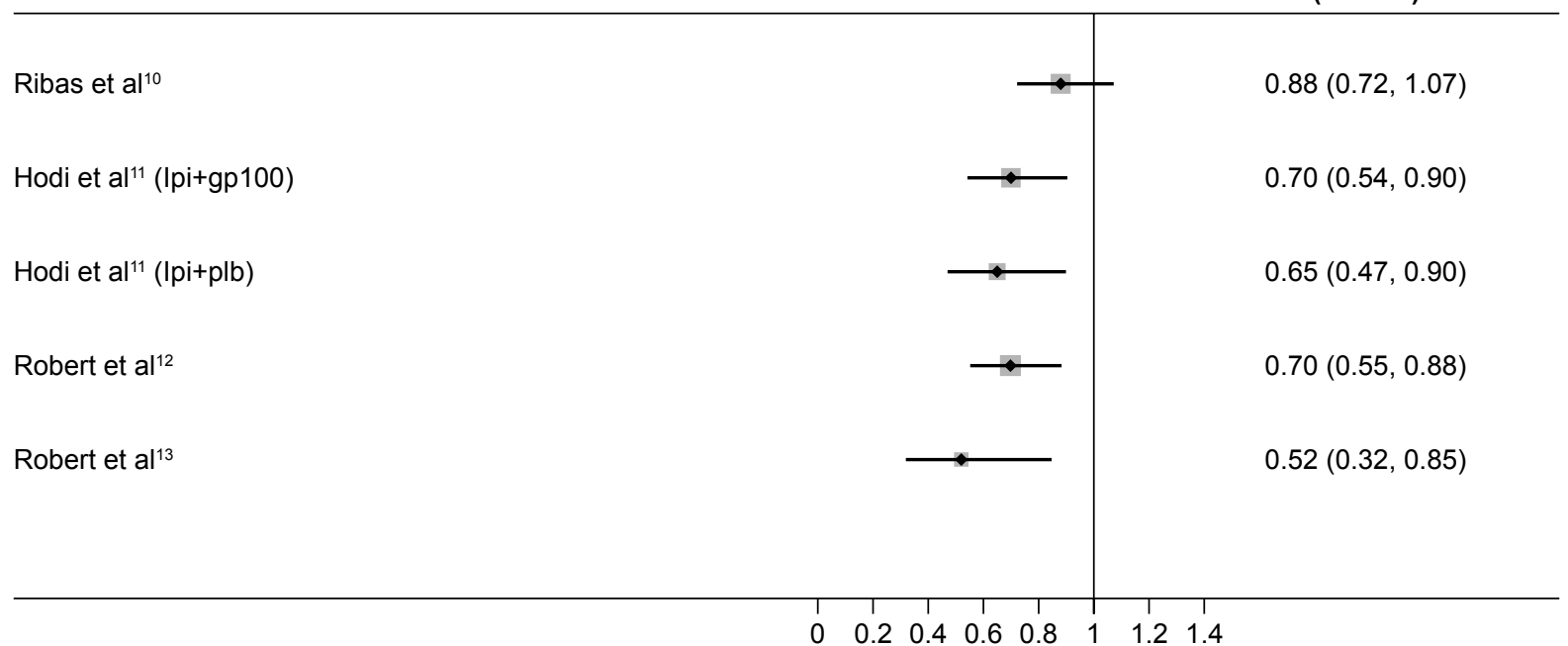

HR $(95 \% \mathrm{Cl})$

Figure 5 Subgroup analysis of younger patients.

Note: Fixed-effects model were used in the analysis.

Abbreviations: HR, hazard ratio; Ipi, ipilimumab; plb, placebo. 
Aging contributes to decreased clonal expansion of $\mathrm{CD} 8^{+}$ effector T cells, hence leading to decreased immunity. ${ }^{16}$ This may have direct influence on the role of PD-1 inhibitors. Evidence ${ }^{17-19}$ shows that aging results in the decline of the functions of DC and T cell and this may reduce the efficacy of ICIs, in particular CTLA-4 inhibitors. ${ }^{15}$

Up-to-date trials indicated a potential efficacy-aging association in immune checkpoint blockade treatments. Scientists in CheckMate-0017 study found that the OS did not favor PD-1 inhibitors in patients aged over 75 years. ${ }^{20}$ On the contrary, PD-1 inhibitor seems to favor melanoma patients aged over 75 . Although this may be caused by the small sample size, our study, with a larger scope and more numbers of patients included, attempted to provide new findings. Another comprehensive review made a comparison of the efficacy of different ICIs in different tumor types generally. ${ }^{21}$ Nevertheless, they did not provide a specific analysis and any specific suggestions for clinicians.

In the field of tumor immunology and immunotherapy, the impact of immunosenescence on ICI effectiveness varies in different cancers. ${ }^{15}$ Interestingly, efficacy of PD-1 inhibitors is totally different in melanoma and NSCLC patients older than 75 years. Our observation indicated that melanoma patients older than 75 years benefit more from nivolumab. On the contrary, among NSCLC patients older than 75 years, there was even no significantly longer OS (HR: 1.02, 95\% CI: $0.35-1.69, P=0.971)$ than controls. Theoretically, immunosenescence can reduce the efficacy of immune-based therapies. Future explorations are needed to give further explanations.

Our study did have some disadvantages. Patients with different types of melanoma were included with different etiologies. In order to minimize this disadvantage, we applied the randomized effect model when the value of heterogeneity was high. Also, comparatively small sample sizes were in the subgroups, and the subgroup findings required more assessments in future RCTs. We could not compare the toxicity of those drugs and evaluate the impact of immunosenescence on the safety of patients. Currently, there are no published data in relation to the immune-related adverse event in people belonging to different age groups, despite its significance in clinical practice. The biological role of immunosenescence-efficacy correlation should be investigated in the near future.

\section{Conclusion}

ICIs significantly prolonged the OS for melanoma patients in both younger and older groups than controls. Anti-PD-1 agents were more efficient in older melanoma patients versus younger patients. ICIs could be used for older melanoma patients. The difference of effectiveness of PD-1 and CTLA-4 inhibitors in younger and older melanoma patients should get noticed in the future clinical practice and guidelines.

\section{Disclosure}

The authors report no conflicts of interest in this work.

\section{References}

1. Pawelec G. Age and immunity: what is "immunosenescence"? Exp Gerontol. 2018;105:4-9.

2. Ventura MT, Casciaro M, Gangemi S, Buquicchio R. Immunosenescence in aging: between immune cells depletion and cytokines up-regulation. Clin Mol Allergy. 2017;15:21.

3. Bolton C, Smith PA. The influence and impact of ageing and immunosenescence (ISC) on adaptive immunity during multiple sclerosis (MS) and the animal counterpart experimental autoimmune encephalomyelitis (EAE). Ageing Res Rev. 2018;41:64-81.

4. Daste A, Domblides C, Gross-Goupil M, et al. Immune checkpoint inhibitors and elderly people: a review. Eur J Cancer. 2017;82:155-166.

5. Wu Y, Ju Q, Qian B, Zhang F, Shi H. The effectiveness of PD-1 inhibitors in non-small cell lung cancer (NSCLC) patients of different ages. Oncotarget. 2017;9(8):7942-7948.

6. Silva IP, Long GV. Systemic therapy in advanced melanoma: integrating targeted therapy and immunotherapy into clinical practice. Curr Opin Oncol. 2017;29(6):484-492.

7. Wu Y, Shi H, Jiang M, et al. The clinical value of combination of immune checkpoint inhibitors in cancer patients: a meta-analysis of efficacy and safety. Int J Cancer. 2017;141(12):2562-2570.

8. O'Reilly A, Larkin J. The safety of nivolumab for the treatment of metastatic melanoma. Expert Opin Drug Saf. 2017;16(8):955-961.

9. Ferrara R, Mezquita L, Auclin E, Chaput N, Besse B. Immunosenescence and immunecheckpoint inhibitors in non-small cell lung cancer patients: does age really matter? Cancer Treat Rev. 2017;60:60-68.

10. Ribas A, Kefford R, Marshall MA, et al. Phase III randomized clinical trial comparing tremelimumab with standard-of-care chemotherapy in patients with advanced melanoma. J Clin Oncol. 2013;31(5):616-622.

11. Hodi FS, O'Day SJ, McDermott DF, et al. Improved survival with ipilimumab in patients with metastatic melanoma. NEngl J Med. 2010; 363(8):711-723.

12. Robert $\mathrm{C}$, Thomas L, Bondarenko I, et al. Ipilimumab plus dacarbazine for previously untreated metastatic melanoma. NEngl JMed. 2011;364(26): 2517-2526.

13. Robert C, Long GV, Brady B, et al. Nivolumab in previously untreated melanoma without BRAF mutation. $N$ Engl J Med. 2015;372(4): 320-330.

14. Ribas A, Puzanov I, Dummer R, et al. Pembrolizumab versus investigator-choice chemotherapy for ipilimumab-refractory melanoma (KEYNOTE-002): a randomised, controlled, phase 2 trial. Lancet Oncol. 2015;16(8):908-918.

15. Elias R, Karantanos T, Sira E, Hartshorn KL. Immunotherapy comes of age: immune aging \& checkpoint inhibitors. J Geriatr Oncol. 2017; $8(3): 229-235$

16. Czesnikiewicz-Guzik M, Lee WW, Cui D, et al. T cell subset-specific susceptibility to aging. Clin Immunol. 2008;127(1):107-118.

17. Grolleau-Julius A, Harning EK, Abernathy LM, Yung RL. Impaired dendritic cell function in aging leads to defective antitumor immunity. Cancer Res. 2008;68(15):6341-6349.

18. Plowden J, Renshaw-Hoelscher M, Gangappa S, Engleman C, Katz JM, Sambhara S. Impaired antigen-induced CD8+ T cell clonal expansion in aging is due to defects in antigen presenting cell function. Cell Immunol. 2004;229(2):86-92. 
19. Guo Z, Tilburgs T, Wong B, Strominger JL. Dysfunction of dendritic cells in aged C57BL/6 mice leads to failure of natural killer cell activation and of tumor eradication. Proc Natl Acad Sci US A. 2014;111(39): 14199-14204.

20. Keating GM. Nivolumab: a review in advanced squamous non-small cell lung cancer. Drugs. 2015;75(16):1925-1934.
21. Nishijima TF, Muss HB, Shachar SS, Moschos SJ. Comparison of efficacy of immune checkpoint inhibitors (ICIs) between younger and older patients: a systematic review and meta-analysis. Cancer Treat Rev. 2016;45:30-37.

\section{Publish your work in this journal}

OncoTargets and Therapy is an international, peer-reviewed, open access journal focusing on the pathological basis of all cancers, potential targets for therapy and treatment protocols employed to improve the management of cancer patients. The journal also focuses on the impact of management programs and new therapeutic agents and protocols on

\section{Dovepress}

patient perspectives such as quality of life, adherence and satisfaction. The manuscript management system is completely online and includes a very quick and fair peer-review system, which is all easy to use. Visit http://www.dovepress.com/testimonials.php to read real quotes from published authors.

\footnotetext{
Submit your manuscript here: http://www.dovepress.com/oncotargets-and-therapy-journal
} 